\title{
A New Approach for the Betterment in Energy-Aware VM Scheduling
}

\author{
Alekhya Orugonda ${ }^{1}$, Dr. V. Kiran Kumar ${ }^{2}$ \\ ${ }^{I}$ Ph.D Research Scholar Department of Computer Science Dravidian University Kuppam \\ ${ }^{2}$ Research Supervisor-Assistant Professor Department of Computer Science Dravidian University Kuppam
}

\begin{abstract}
Cloud Computing is dealing with an growing interest nowadays as it is present in many purchaser home equipment through advertising the illusion of countless resources in the direction of its clients. Nevertheless it increases intense problems with electricity intake: the better degrees of nice and availability require irrational strength expenses. A digital machine scheduling technique for decreasing strength consumption of IaaS datacenters is required. We need to design a CloudSim-based simulation environment, and implemented actual-international lines for the experiments. We need to show that enormous savings can be accomplished in strength intake with brand new proposed algorithms. Cloud computing is a digital pool of resources which might be supplied to customers through Internet. Cloud computing provider vendors one of the desires is to apply the assets successfully and advantage most profit. This results in task scheduling as a core and challenging difficulty in cloud computing. In this studies a dialogue closer to the useful resource management of digital machines in cloud and a way to make assets extra efficiently available to customers is furnished. The most important focus is on process scheduling. In this present work, a parametric analysis is finished to discover the requirement of technique migration and based totally in this evaluation the migration can be completed on those tactics. . The effectiveness of the paintings is diagnosed in terms of successful execution of the processes inside the deadlines.
\end{abstract}

Keywords: Virtual machines, Scheduling, and Migration, Energy aware

\section{Introduction}

Cloud computing is one of the maximum explosively increasing technologies in the computing enterprise nowadays. However it is critical to understand where it got here from, that allows you to determine out where it'll be heading within the destiny. While there may be no clean reduce evolutionary course to Clouds, many agree with the principles originate from precise areas: Grid Computing and Web 2.0. Grid computing, in its practical form, represents the concept of connecting or more spatially and administratively diverse clusters or supercomputers collectively in a federating manner. The term "the Grid" turned into coined in the mid 1990's to symbolize a huge disbursed structures infrastructure for superior clinical and engineering computing issues. Grids purpose to enable applications to harness the total capacity of resources through coordinated and controlled resource sharing by using scalable virtual businesses. While not all of those principles deliver over to the Cloud, to manipulate, federation, and dynamic sharing of resources is conceptually similar to inside the Grid. This is printed with the aid of, as Grids and Clouds are compared at a summary stage and many ideas are remarkably similar. From a scientific perspective, the desires of Clouds and Grids also are comparable. Both systems try and provide massive quantities of computing power by using leveraging a large number of sites walking diverse packages simultaneously in symphony. The only substantial differences among Grids and Clouds exist in the implementation information, and the reproductions of them. The other major thing, Web 2.0, is also a particularly new idea in the history of Computer Science. The term Web 2.0 turned into at first coined in 1999 in a futuristic prediction by Dracy DiNucci : "The Web we know now, which loads into a browser window in essentially static screen fulls, is most effective an embryo of the Web to come. The first glimmerings of Web 2.Zero are starting to seem, and we're simply beginning to see how that embryo would possibly develop. The Web can be understood now not as screen fuls of text and pics however as a transport mechanism, the ether through which interactivity takes place. It will seem to your computer display screen, for your TV set your vehicle dashboard your cell smartphone handheld recreation machines maybe even your microwave oven." Her vision began to shape, as illustrated in 2004 by the O'Riley Web 2.0 convention, and in view that then the time period has been a pivotal buzz phrase amongst the internet. While many definitions had been furnished, Web 2.0 without a doubt represents the transition from static HTML to harnessing the Internet and the Web as a platform in of itself. Web 2.0 gives a couple of stages of software services to customers throughout the Internet. In essence, the net turns into an application suite for users. Data is outsourced to anyplace it's far wanted, and the customers have general manage over what they interact with, and spread hence. This calls for large, dynamic and scalable website hosting sources for these programs. This demand gives the user-base for a whole lot of the commercial Cloud computing enterprise 
today. Web 2.0 software program calls for abstracted resources to be allocated and relinquished at the fly, relying on the Web's visitors and service utilization at every web page.

Furthermore, Web 2.0 brought Web Services requirements and the Service Oriented Architecture (SOA) which define the interaction between customers and cyber infrastructure. In summary, Web 2.Zero defined the interplay standards and person base, and Grid computing described the underlying infrastructure capabilities. A Cloud computing implementation normally allows users immigrate their information and computation to a remote region with minimal impact on device performance. This presents a number of benefits which couldn't otherwise be found out. These advantages encompass:

- Scalable - Clouds are designed to supply as lots computing strength as any consumer needs. While in practice the underlying infrastructure is not endless, the cloud assets are projected to ease the developer's dependence on any precise hardware.

- Quality of Service (QoS) - Unlike preferred statistics centers and advanced computing sources, a nicely-designed Cloud can task a far higher QoS than traditionally feasible. This is because of the dearth of dependence on precise hardware, so any bodily gadget disasters may be mitigated without the prerequisite person focus.

- Specialized Environment - Within a Cloud, the person can utilize customized equipment and services to satisfy their needs. This may be to make use of the cutting-edge library, toolkit, or to support legacy code within new infrastructure.

- Cost Effective - Users finds simplest the hardware required for each venture. This reduces the hazard for establishments probably want construct a scalable system, thus offering more flexibility, since the user is most effective buying wished infrastructure at the same time as retaining the option to growth services as needed in the future.

- Simplified Interface - Whether the usage of a selected software, a hard and fast of gear or Web services, Clouds offer get admission to a probably great amount of computing sources in an easy and user-centric manner. We have investigated such an interface within Grid systems through using the Cyber aide challenge. Many of the features cited above outline what Cloud computing can be from a user angle. However, Cloud computing in its physical shape has many unique meanings and bureaucracy. Since Clouds are defined by the offerings they provide and now not via applications, an included as-a service paradigm has been described to demonstrate the numerous ranges within an ordinary Cloud.

Cloud computing includes many components of sharing software program and hardware answers, along with computing and storage sources, utility runtimes or complex application functionalities. The cloud paradigm modified the manner people study computing infrastructures. First, one does no longer want to be professional in infrastructure administration, operation and maintenance even supposing huge scale systems are utilized. Second, the elasticity of Infrastructure as a Service clouds allows these structures to higher observe the users' real needs. However, there is also an adversary impact: the virtualized nature of those structures detaches users from several operational issues like strength green utilization that has been addressed previously in the context of parallel and allotted structures, and in large part stays un noticed. The Cloud computing generation made a qualitative step forward as its far present in many consumer home equipment along with numerous mobile devices. They advertise the illusion of endless sources toward the consumers, meanwhile it additionally increases intense issues with strength consumption: the higher stages of fine and availability require irrational strength fees, according to a few specialists the power of assets spent for idling constitutes an extensive quantity. Current trends are claimed to be genuinely unsustainable with appreciate to useful resource utilization, $\mathrm{CO} 2$ footprint and over all power efficiency. It is predicted that in addition increase is confined with the aid of strength consumption, in addition extra competitiveness of groups are and can be strongly tied to these issues. As cloud services become an increasing number of popular, small- and medium-sized cloud carrier providers will quickly face increasing consumer demands that cannot be met with their modern-day infrastructures. These consumer demands range from occasional wishes for extreme quantity of resources (in comparison to the issuer's cutting-edge infrastructure) to the need for multi-web page digital system deployment options that permit enhanced services which include catastrophe recovery. Thus those providers need to growth the scale in their infrastructure by using introducing more than one datacenters overlaying numerous locations, and supplying unprecedented quantity of assets. Current IaaS solutions offer the opportunity for service carriers to fulfill those needs by way of focusing their interest to non-technical issues just like the increased working value in their datacenters. Despite power consumption is a main component of those working expenses, modern IaaS answers slightly handle the infrastructure with strength aware solutions. Therefore providers were confined to lessen their consumption on the hardware level to this point, independently from the carried out IaaS solution. Energy costs also are increasing, and datacenter device is stressing electricity and cooling infrastructures, for this reason the primary problem is not the present day quantity of statistics center emissions however the fact that these emissions are raising quicker than another carbon emission. Although these improvements in 
hardware are important, we believe that the energy consumption can also be significantly decreased with software program in over-provisioned IaaS systems. Over-provisioning is a key conduct at smaller sized providers, who provide offerings for users with occasional peaks in useful resource needs.

Reducing the carbon footprint of European international locations is likewise a ought to and anticipated by means of the European Commission, in addition to boom the quantity and size of European Cloud carriers .By federating those vendors, extra competitive initiatives can be founded, that may be sophistically controlled to meet those expectancies. The widespread purpose of the management layer in a Cloud federation is to distribute load most of the collaborating cloud vendors, to decorate user pride through filtering out underperforming providers, and schedule and execute service calls with minimized strength consumption inside the selected IaaS gadget. To attain this, we've already proposed a structure called Federated Cloud Management. In this holistic method a two-stage brokering solution is used: a meta-brokering thing is used to direct carrier calls to carriers, after which a cloud-brokering aspect to map these calls onto an optimized quantity of virtual machines. While Cloud computing is converting IT infrastructure, it additionally has had a drastic effect on Distributed Systems itself. Gone are the IBM Mainframes of the eighty's which ruled the employer landscape. While some mainframes still exist, they may be used best for batch associated processing obligations and are pretty unused for clinical packages as they may be inefficient at Floating Point Operations. As such, they have been changed with Beowulf Clusters of the 90's and 00's. The novelty of Supercomputing is that instead of just one huge system, many machines are linked together and used to obtain a not unusual intention, thereby maximizing the overall pace of computation. Clusters constitute a greater commodity based supercomputer, where off the shelf CPUs are used in place of the surprisingly customized and expensive processors in Supercomputers. Supercomputers and Clusters are high-quality acceptable for large scale programs along with particle physics, weather forecasting, weather studies, molecular modeling, bioinformatics, and physical simulations, to call some. These applications are regularly termed "Grand Challenge" packages and represent most of the people of clinical calculations finished on Supercomputing assets today. However, currently these medical Clusters and Supercomputers are being subverted by the Cloud computing paradigm itself. Many scientists are figuring out the energy that Clouds provide on demand, and are looking to harness the uncooked capability for their very own desires without addressing the daunting undertaking of going for walks their personal Supercomputer. While many manufacturing-stage Grid computing systems have looked to provide similar offerings for the past 10 years through offerings which includes the Open Science Grid and TeraGrid, the fulfillment of such Grid systems has been mixed. Currently we're on the cusp of a merger between the distributed Grid middleware and contemporary Cloud technology inside the realm of medical computing. Recent tasks together with the NSF Future Grid Project and the DOE Magellan Project goal to leverage the advances of Grid computing with the brought benefits of Clouds (mentioned in element in Chapter 2). Yet these projects are at a research-only degree and of their infancy; the achievement of Cloud computing inside the medical community is still yet to be decided.

\section{Motivation for this Work}

As new distributed computing technologies like Clouds become an increasing number of famous, the dependence on electricity also will increase. Currently it's far expected that statistics centers eat 0.5 percent of the sector's general electricity usage. If modern demand continues, its miles projected to quadruple by using 2020. In 2005, the entire energy consumption for servers and their cooling units become projected at $1.2 \%$ the overall U.S. Electricity intake and doubling each 5 years. The majority of the electricity utilized in now-a-day's society is generated from fossil fuels which produce harmful $\mathrm{CO} 2$ emissions. Therefore, its miles imperative to decorate the performance and capability sustainability of big data facilities. One of the essential components of virtualization technologies mired in Cloud environments is aid consolidation and control. Using hypervisors within a cluster surroundings lets in for some of standalone bodily machines to be consolidated to a virtualized surroundings, thereby requiring less physical resources than ever earlier than. While this improves the state of affairs, it frequently is inadequate. Large Cloud deployments require heaps of physical machines and megawatts of electricity. Therefore, there is a want to create an efficient Cloud computing system that utilizes the strengths of the Cloud even as minimizing its strength and environmental footprint. In order to correctly and absolutely unify a Green issue to the following generation of Distributed Systems, a fixed of guidelines is wanted. These guidelines should constitute a route of sustainable development that can be integrated into information center construction and control as an entire. While the framework supplied on this paper represents many promising methods to lessen power consumption, genuine sustainable development additionally relies upon on locating a renewable and reliable energy source for the information middle itself. When combined, a lot of these day's limits inside the length of information facilities will begin to deteriorate. 


\section{Environmental Concerns}

While the monetary expenses of running the world's information facilities can be extremely astonishing as a whole, there is also some other essential attention; the Environment. As proven in figure 1.1, almost $70 \%$ of the U.S.'s electricity comes from non-renewable fossil fuels. As contemporary records middle strength intake is estimated at 2.Four\%, the general $\mathrm{CO} 2$ emissions because of the statistics centers represents sobering reality to the environmental effect the enterprise has created. To well deal with the sustainability of records facilities, it'd make experience to recognition often at the assets of strength. However accomplishing the goal of producing $70 \%$ of the U.S.'s cutting-edge electricity generation from renewable power resources is a challenge so that it will take at least a era, if not many generations. In the intervening time, it is vital to recognition at the records centers themselves to improve performance, no longer best for economic motives, however additionally for the environment. Therefore, improving the energy efficiency inside records centers does not look to revolutionize their sustainability, however alternatively to improve upon an already present infrastructure. This paintings isn't supposed to take away the need for renewable sources, however as a substitute to try to alleviate the burdening power needs during this lengthy transition duration away shape fossil fuels. As described with the aid of Thomas Seager's Sustainability Spectrum, the work brought in this manuscript lies in the Reliability section of sustainability. Nevertheless, its miles essential to make what power performance upgrades we will that allows you to reduce the worldwide climate effect. Cloud computing additionally makes experience environmentally from a whole inter-degree standpoint. Much of what Cloud computing represents is the consolidation of assets from an intra perspective to an inter standpoint, thereby pushing statistics and offerings from character websites to a greater centralized system. While there are various scaling and privateness problems Cloud computing need to address, the blessings are clean from an environmental angle. Typically each individual statistics middle site is fairly small in length and could therefore have greater conventional means of cooling. These small records center cooling systems are regularly just huge air conditioning setups that result in a very high facts middle Power Usage Effectiveness (PUE), ensuing in greater energy used universal. Many commercial Cloud deployments offer a big scale facts center operations. This lets in every website to be take advantage of the economies of scale which aren't available to smaller-sized records facilities. These larger Cloud centers can be capable of enforce advanced cooling solutions which preserve the PUE low, together with superior chiller towers or superior water-cooling setups. Currently, Google has applied some of these massive records centers and is attaining a PUE price between 1.1 and 1.Three, compared to the countrywide common of two.1. If the IT industry keeps to shift the general computing sources from many small information centers to a smaller range of large facts centers, the cooling savings on my own will have a drastic impact on the environment, just by means of taking gain of the fundamental principles of scaling.

\section{Related Work}

Regarding power performance in a single cloud, Cioara et al. [Cioara et al., 2011] delivered an energy conscious scheduling coverage to consolidate strength management by means of the use of reinforcement gaining knowledge of techniques to restore a provider middle to a power green state. Feller et al. Proposed a dynamic cluster supervisor referred to as Snooze [Feller et al., 2010], that's capable of dynamically consolidate the workload of a heterogeneous massive-scale cluster composed of re- sources the usage of virtualization. In a later paintings [Feller et al., 2012], they use strength meters to display electricity utilization of cloud resources, and estimate the resource usage of VMs. Their mechanisms cope with VM placement, relocation and migration by means of keeping VMs on as few nodes as viable. Cardosa et al. [Cardosa et al., 2009] presented a unique suite of techniques for placement and power consolidation of VMs in datacenters taking gain of the min-max and stocks functions inherent in virtualization technologies, like VMware and Xen. These capabilities permit to specify the minimal and maximum amount of re- assets that can be allotted to a VM, and pro- vide a shares based mechanism for the hypervisor to distribute spare assets among contending VMs. Lee et al. [Lee et al., 2010] speak service request scheduling in Clouds based totally on viable income. They suggest a pricing model the usage of processor sharing for composite services in Clouds. Lucas-Simarro et al. [Lucas-Simarro et al., 2013] proposed oneof-a-kind scheduling techniques for superior deployment of services throughout more than one clouds based totally on diverse optimization standards. The tested scheduling guidelines encompass finances, overall performance, load balancing and other dynamic conditions, but they unnoticed energy efficiency, that is the goal of our work. Regarding fuzzy methods, Salleh et al. [Salleh et al., 1999] have proven how to set up and use fuzzy common sense in a conventional way for dynamic venture scheduling in multiprocessor systems. We have already published a paper [Dombi and Kert_esz, 2011] on making use of the pliant method to process scheduling in Grids. In this contemporary paper we would really like to show that it's also possible to apply pliant device for scheduling, with just a few policies. The novelty of this contribution lies within the manner we practice the pliant machine to Clouds: the manner we choose cloud-unique residences as parameters of the pliant gadget. Concerning cloud simulations, Berge et al. [vordem Berge et al., 2012] have 
designed a simulator referred to as SVD within the Cool Em All mission for investigating power intake in datacenters. It is an extended model of the GSSIM simulator, and they're making plans to help software modeling and profiling via benchmarks. Regarding federation-huge simulations, Sotiriadis et al. [Sotiriadis et al., 2013] investigated inter- cloud simulations by using growing the SimIC simulation toolkit this is capable of mimic the inter cloud provider formation to allow the research of carrier-oriented cloud utilization, but they forget strength efficiency.

\section{Simulation for Cloud Computing}

We can use the CloudSim simulator to broaden a simulation environment for our research. Beloglazov and Buyya have al- ready commenced to look at how electricity performance could be investigated inside this simulator. Data- centers devour huge quantities of electricity ensuing in excessive operating fees and multiplied carbon dioxide emissions. The dynamic consolidation of VMs using live migration and switching on idle nodes can be used to optimize resource utilization and reduce electricity consumption, however they argue that competitive consolidation can also lead to performance degradation. They proposed adaptive heuristics for dynamic consolidation of VMs primarily based on an evaluation of historical information from the resource usage by means of VMs, even as making sure a high stage of adherence to the Service Level Agreements (SLA).

\section{Proposed Work}

There are an increasing number of Cloud Services available inside the Internet. Cloud offerings may be a thing of a machine and unique Cloud Servers that might offer unique offerings. In this present paintings we have defined a couple of cloud surroundings. Each cloud server is defined with sure limits in phrases of reminiscence and the CPU specs. Now because the customers input to the system, the person request is done in phrases of strategies. To represent the parallel consumer requests, $\mathrm{n}$ wide variety of requests are been generated by the customers. All these requests are to be handled by the cloud servers in parallel via the usage of the multiple clouds idea. A middle layer is defined among the cloud servers and the clients' requests a good way to carry out the allocation of the tactics to unique clouds under overload and below load situations. As person requests are completed, a few parameters also are defines with each requests. These parameters are procedure time, deadline, input output specifications etc. In the overall case, the allocation of the tactics is finished in a sequential order. Each process ought to be accomplished inside the cut-off date restrict. But if a couple of processes occur at equal time and not get performed before the cut-off date, in such case the tactics is switched from one cloud server to different called the manner migration. In this gift work, a parametric evaluation is accomplished to discover the requirement of system migration and based totally in this analysis the migration can be accomplished on these processes. The effectiveness of the paintings is diagnosed in terms of successful execution of the procedures in the closing dates.

\section{Research Design}

The proposed machine is center layer structure to carry out the cloud allocation in case of underneath load and overload conditions. The over load conditions may be treated by means of using the standards of procedure migration. The middle layer will exist between the clouds and the clients. As the request might be completed by using the consumer this request can be accepted by way of the middle layer and the analysis of the cloud servers is carried out by means of this middle layer. The middle layer is responsible for three major responsibilities.

1. Scheduling the consumer requests.

2. Monitor the cloud servers for its abilities and to perform the method allocation.

3. Process Migration in overload situations.

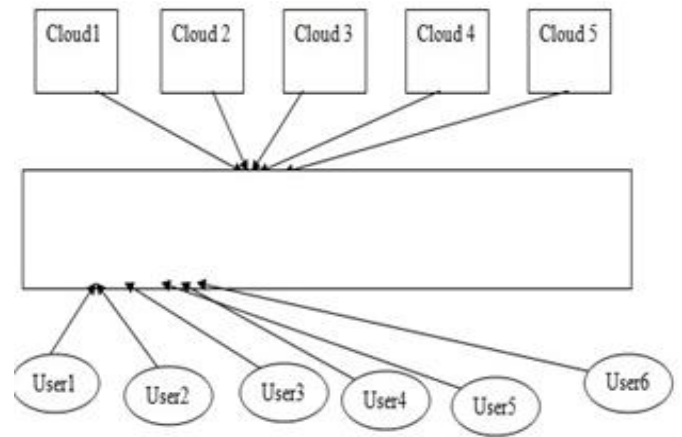

Fig 1: Clouds and Users 


\section{Algorithm}

Input M number of Clouds with L number of Virtual Machines associated with each cloud.

Define the available memory and load for each virtual machine.

Assign the priority to each cloud.

Input $\mathrm{N}$ number of user process request with some parameters specifications like arrival time, process time, required memory etc.

Arrange the process requests in order of memory requirement

For $\mathrm{i}=1$ to $\mathrm{N}$

\{

Identify the priority Cloud and Associated VM having load < Capacity

Perform the initial allocation of process to that particular VM and the

Cloud

\}

For $\mathrm{i}=1$ to $\mathrm{N}$

\{

Identify the Free Time slot on priority cloud to perform the allocation. As the free slot identify, record the start time, process time, turnaround time and the deadline of the process.

\}

For $\mathrm{i}=1$ to $\mathrm{N}$

\{

If finish time (process (i)) >Deadline (Process (i))

\{

Print "Migration Required"

Identify the next high priority cloud that having the free memory and the time slot and perform the migration of the process to that particular cloud and the virtual machine.

\}

\}

The input taken here includes the number of clouds in the environment, number of VMs supported by each cloud, memory availability, IO availability for each virtual machine. By using these parameters the clouds server is configured.

\begin{tabular}{|l|l|l|l|l|l|l|}
\hline USER ID & $\begin{array}{l}\text { ARRIVAL } \\
\text { TIME }\end{array}$ & $\begin{array}{l}\text { PROCESS } \\
\text { TIME }\end{array}$ & DEADLINE & $\begin{array}{l}\text { INPUTOUTPUT } \\
\text { REQUEST }\end{array}$ & $\begin{array}{l}\text { MEMORY } \\
\text { REQUEST }\end{array}$ & $\begin{array}{l}\text { SERVICE } \\
\text { TYPE }\end{array}$ \\
\hline 1 & 8 & 5 & 15 & 5 & 29755 & 0 \\
\hline 2 & 95 & 10 & 111 & 4 & 3955 & 0 \\
\hline 3 & 90 & 9 & 110 & 2 & 1250 & 1 \\
\hline 4 & 91 & 8 & 111 & 3 & 12555 & 1 \\
\hline 5 & 16 & 7 & 25 & 1 & 1475 & 1 \\
\hline 6 & 85 & 8 & 95 & 5 & 1100 & 0 \\
\hline 7 & 36 & 6 & 55 & 2 & 15500 & 0 \\
\hline 8 & 65 & 2 & 75 & 3 & 10890 & 1 \\
\hline 9 & 15 & 8 & 25 & 2 & 22350 & 1 \\
\hline 10 & 20 & 6 & 30 & $1: C 250$ & 1 \\
\hline
\end{tabular}

Table 1: Cloud Computing Parameters

Table 1. List of parameters: Table 1 shows the values of Arrival time, Process time, Deadline, I/O request, mem request and Type of service are computed to schedule requests of users.

Table: 2: List of Para meters in sorted Order according to the Memory

\begin{tabular}{|l|l|l|l|l|l|l|}
\hline USER ID & $\begin{array}{l}\text { ARRIVA } \\
\text { L TIME }\end{array}$ & $\begin{array}{l}\text { PROCES } \\
\text { S TIME }\end{array}$ & DEADLIN E & $\begin{array}{l}\text { INPUTOUTPUT } \\
\text { REQUEST }\end{array}$ & $\begin{array}{l}\text { MEMORY } \\
\text { REQUEST }\end{array}$ & $\begin{array}{l}\text { SERVIC } \\
\text { E TYPE }\end{array}$ \\
\hline 5 & 85 & 7 & 95 & 4 & 1100 & 0 \\
\hline 3 & 90 & 8 & 110 & 3 & 1150 & 0 \\
\hline 6 & 15 & 6 & 25 & 1 & 1475 & 1 \\
\hline 3 & 95 & 11 & 110 & 3 & 4535 & 1 \\
\hline 8 & 15 & 3 & 25 & 3 & 109800 & 1 \\
\hline 5 & 90 & 6 & 105 & 3 & 12555 & 0 \\
\hline 6 & 38 & 8 & 50 & 0 & 15672 & 0 \\
\hline 7 & 65 & 8 & 80 & 1 & 21750 & 1 \\
\hline 9 & 22 & 3 & 35 & 3 & 22370 & 1 \\
\hline 1 & 10 & 2 & 15 & 2 & 30870 & 1 \\
\hline
\end{tabular}




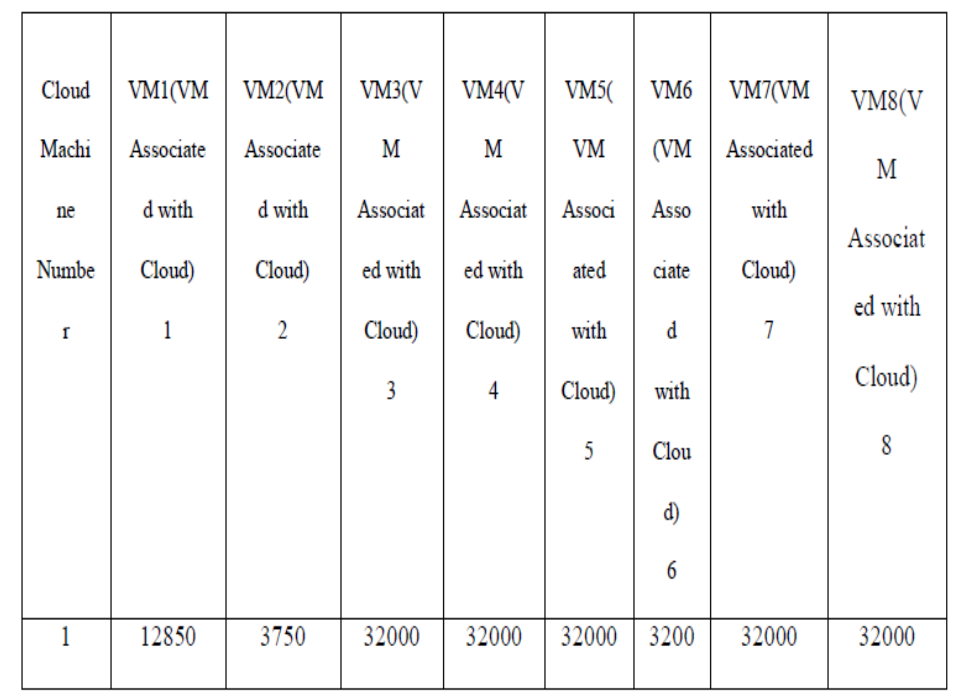

\begin{tabular}{|c|c|c|c|c|c|c|c|c|}
\hline & 32000 & 32000 & 31900 & 31900 & 31900 & 3191 & 31900 & 31910 \\
\hline 2 & 31900 & 31900 & 31901 & 31902 & 31902 & 3190 & 31902 & 31902 \\
\hline 3 & 31902 & 31910 & 31922 & 31922 & 31900 & 3191 & 31900 & 31900 \\
\hline 4 & 31900 & 31908 & 32010 & 32000 & 32000 & 3200 & 32000 & 32000 \\
& & & & & & 9 & & \\
\hline 5 & 3200 & 32000 & 32003 & 32000 & 3600 & 3200 & 32000 & 32000 \\
\hline & & & & & & 7 & & \\
\hline
\end{tabular}

Table: 3 Arailable Memory After allocation

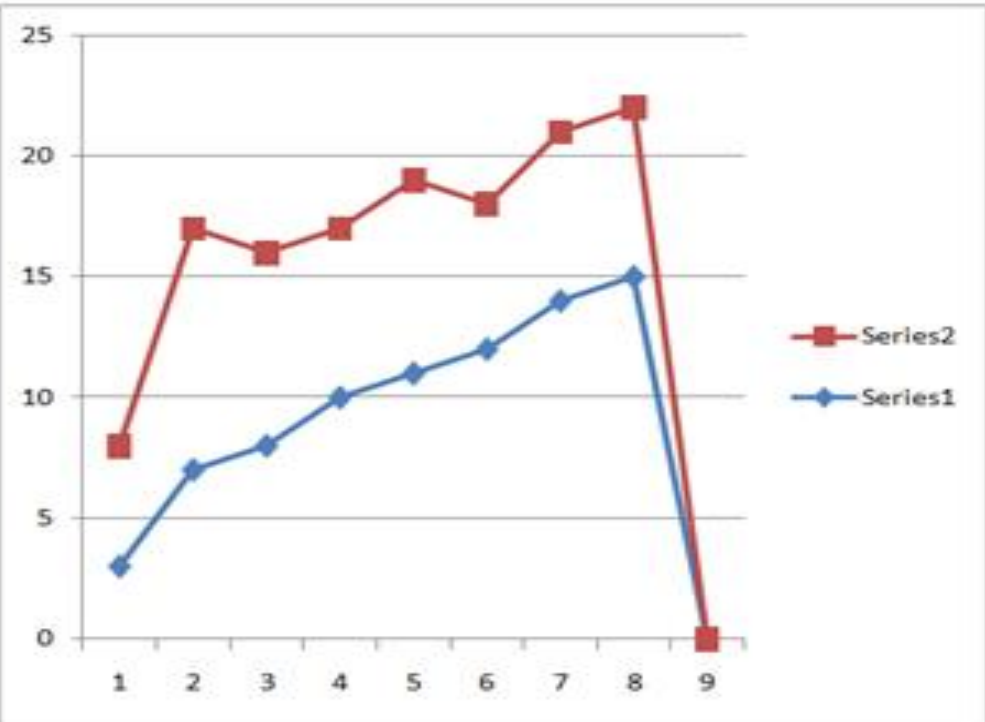

Fig 1: Taking Time (in Seconds) on Y-axis, Process and Requests from the User on X- Axis 


\section{Limitations}

The presented work is about to perform the scheduling and the allocation of the processes to the clouds in case of under load and overload conditions. In case of over load condition, the migration of the processes is performed from one cloud to other. The Future enhancement of the work is possible in the following directions.

1. The presented work is defined the overload conditions in terms of deadline as well as the memory limit of the Clouds. In future some other parameters can also be taken to decide the migration condition.

2. The presented work is defined for the public Cloud environment, but in future, the work can be extended to private and the hybrid Cloud environment.

\section{Conclusion}

Cloud Computing is facing a growing attention nowadays and it raises intense issues with energy consumption: the higher ranges of fine and availability require irrational power prices. Reducing the carbon footprint of European countries is also a should and anticipated by way of the European Commission, as well as to increase the wide variety and size of European Cloud vendors. a resource allocation scheme on multiple Clouds in both the under load and the over load conditions is provided. As the request is performed by the user, certain parameters are defined with each user request, these parameters includes the arrival time, process time, deadline and the input output requirement of the processes. The Cloud environment taken in this work is the public cloud environment with multiple clouds. Each Cloud is here defined with some virtual machines. The obtain results shows the successful execution of all the processes within time limit. The work is performed on a generic system that can have n number of Clouds.

\section{References}

[1]. Anthony T.Velte, Toby J.Velte, Robert Elsenpeter, "Cloud Computing Practical Approach". Xun Xu. "From Cloud Computing to cloud".

[2]. Jeongseob Ahn Changdae Kim, Jaeung Han, Young-ri Choit, And Jaehyuk Huh, "Dynamic Virtual Machine Scheduling In clouds For Architectural Shared Resources", 2011 IEEE manufacturing”,2011 Elesevier Ltd.

[3]. Zheng Hu, Kaijun Wu, Jinsong Huang,"An Utility-Based Job Scheduling Algorithm for Current Computing Cloud Considering Reliability Factor", 2012 IEEE.

[4]. Masaya Yamada Yuki Watanabe, Saneyasu Yamaguchi,”AN Integrated 1/0 Analyzing System for Virtualized Environment, 2011.

[5]. AshutoshIngole ,SumitChavan, UtkarshPawde "An Optimized Algorithm for Task Scheduling based on Activity based Costing in Cloud Computing", 2nd National Conference on Information and Communication Technology (NCICT) 2011 Proceedings published in International Journal of Computer Applications® (IJCA) .

[6]. Daniel Warneke, Odej Kao "Exploiting Dynamic Resource Allocation for Efficient Parallel Data Processing in the Cloud" IEEE Transactions on Parallel and Distributed Systems.

[7]. SujitTilak, Prof. DiptiPatil, "A Survey of Various Scheduling Algorithms in Cloud Environment", and International Journal of Engineering Inventions ISSN: 2278-7461, www.ijeijournal.com Volume 1, Issue 2 (September 2012) PP: 36-39.

[8]. SandeepTayal "Tasks Scheduling optimization for the Cloud Computing Systems", International Journal of Advanced Engineering Sciences and Technologies Volume No.5, Issue No. 2, $111-115$

[9]. P. Barham, B. Dragovic, K. Fraser, S. Hand, T. L. Harris, A. Ho,R. Neugebauer, I. Pratt, and A. Warfield. Xen and the art of virtualization. In Proceedings of the 19th ACM Symposium on Operating Systems Principles, pages 164-177, New York, U. S. A., Oct. 2003.

[10]. K.J. Barker, K. Davis, A. Hoisie, D.J. Kerbyson, M. Lang, S. Pakin, and J.C. Sancho. Entering the petaflop era: the architecture and performance of Roadrunner. In Proceedings of the 2008 ACM/IEEE conference on Supercomputing. IEEE Press Piscataway, NJ, USA, 2008

[11]. Christian Belady. The Green Grid Data center Efficiency Metrics: PUE and DCIE. Technical report, The Green Grid, Feb. 2007.

[12]. T.R. Bird. Methods to improve bootup time in Linux. In Proc of the Ottawa Linux Symp, 2004.

[13]. Deva Bodas. Data Center Power Management and Benefits to Modular Computing. In Intel Developer Forum, 2003.

[14]. Rajkumar Buyya, Chee Shin Yeo, and Srikumar Venugopal.Market-oriented cloud computing: Vision, hype, and reality for delivering it services as computing utilities. In Proceedings of the 10th IEEE International Conference on High Performance Computing and Communications (HPCC-08, IEEE CS Press, Los Alamitos, CA, USA), pages 5-13, 2008.

[15]. C. Catlett. The philosophy of TeraGrid: building an open, extensible, distributed TeraScale facility. In 2nd IEEE/ACM International Symposium on Cluster Computing and the Grid, 2002, pages 8-8, 2002.

[16]. CERN. LHC Computing Grid Project. Web Page, December 2003.

[17]. JS Chase, DE Irwin, LE Grit, JD Moore, and SE Sprenkle. Dynamic virtual clusters in a grid site manager. In 12th IEEE International Symposium on High Performance Distributed Computing, 2003. Proceedings, pages 90-100, 2003.

[18]. J.J. Dongarra, H.W. Meuer, and E. Strohmaier. Top 500 supercomputers. Website, November 2008.

[19]. [20] W. Feng and K.W. Cameron. The Green500 List: Encouraging Sustainable Supercomputing. Pages 50-55. IEEE Computer Society, 2007.

[20]. W. Feng, A. Ching, C.H. Hsu, and V. Tech. Green Supercomputing in a Desktop Box. In IEEE International Parallel and Distributed Processing Symposium, 2007. IPDPS 2007, pages 1-8, 2007.

[21]. W. Feng, X. Feng, and R. Ge. Green Supercomputing Comes of Age. IT PROFESSIONAL, 10(1):17, 2008.

[22]. J. Fontan, T. Vazquez, L. Gonzalez, R. S. Montero, and I. M. Llorente. OpenNEbula: The Open Source Virtual Machine Manager for Cluster Computing. In Open Source Grid and Cluster Software Conference, San Francisco, CA, USA, May 2008.

[23]. William Forrest. How to cut data centre carbon emissions? Website, December 2008.

[24]. I. Foster and C. Kesselman. Globus: A Metacomputing

[25]. Infrastructure Toolkit. International Journal of Supercomputer Applications, 11(2):115-128, 1997. $\mathrm{ftp}: / / \mathrm{ftp}$.globus.org/pub/globus/papers/globus.pdf.

[26]. I. Foster, C. Kesselman, et al. The Physiology of the Grid: An Open Grid Services Architecture for Distributed Systems Integration. Technical report, Argonne National Laboratory, Chicago, January 2002.

[27]. I. Foster, C. Kesselman, and S. Tuecke. The Anatomy of the Grid: Enabling Scalable Virtual Organizations. Intl. J. Supercomputer Applications, 15(3), 2001.

[28]. Anton Beloglazov, JemalAbawajy, RajkumarBuyya "Energy-aware resource allocation heuristics for efficient management of data centers for Cloud computing", Future Generation Computer Systems 28 (2012) 755-768. 